\title{
CORRELAÇÕES ENTRE AS PROPRIEDADES DA MADEIRA E DO CARVÃO VEGETAL DE HÍBRIDOS DE EUCALIPTO ${ }^{1}$
}

\author{
Vássia Carvalho Soares², Maria Lúcia Bianchi ${ }^{3}$, Paulo Fernando Trugilho ${ }^{3}$, Ariclene Júnior Pereira ${ }^{4}$ e \\ Juliana Höfler ${ }^{5}$
}

\begin{abstract}
RESUMO - Foram estudadas madeiras de híbridos de Eucalyptus grandis x Eucalyptus urophylla, em três idades diferentes, com o objetivo de avaliar o comportamento da madeira e do carvão vegetal produzido diante do aumento controlado de temperatura, bem como verificar a influência das características da madeira sobre o carvão vegetal. Foram realizadas análises químicas (extrativos totais, lignina, holocelulose, celulose, cinza e análise elementar) e térmicas (análise termogravimétrica - TG, análise térmica diferencial - DTA e calorimetria $1,67^{\circ} \mathrm{C} \mathrm{min}{ }^{-1}$ e temperatura final de $450^{\circ} \mathrm{C}$. No carvão vegetal produzido, foram realizadas análise imediata, elementar e térmica. A densidade básica da madeira correlacionou-se positivamente com a relação carbono/ hidrogênio $(\mathrm{C} / \mathrm{H})$ e negativamente com os teores de cinza, nitrogênio, oxigênio, enxofre e relação siringil/ guaiacil (S/G). Os teores de cinza, nitrogênio, enxofre e S/G da madeira correlacionaram-se positivamente entre si e negativamente com a variável C/H da madeira. O teor de carbono fixo (TCF), o poder calorífico do carvão vegetal, o teor de carbono elementar e a relação $\mathrm{C} / \mathrm{H}$ correlacionaram-se positivamente entre si e negativamente com o teor de materiais voláteis (TMV). O teor de lignina correlacionou-se positivamente com o rendimento gravimétrico em carvão (RGC) e negativamente com o TCF.
\end{abstract}

Palavras-chave: Carbonização; Eucalipto; Lignocelulósico.

\section{CORRELATIONS BETWEEN THE PROPERTIES OF EUCALYPTUS HYBRIDS WOOD AND CHARCOAL}

\begin{abstract}
Three different ages of Eucalyptus grandis $x$ Eucalyptos urophylla wood hybrids were studied, with purpose of evaluating wood and charcoal behavior by temperature rising, and investigating the influence of wood characteristics on charcoal. Wood was analyzed chemically (total extractives, lignin, holocellulose, cellulose, ash and elementary composition) and thermally (thermogravimetry analysis - TG, differential thermal analysis - DTA and differential scanning calorimetry - DSC). Also, basic density and higher heating value were evaluated. Charcoal was produced in muffle furnace with constant heating rate of $1,67^{\circ} \mathrm{C} \mathrm{min}^{-1} \mathrm{up}$ to $450^{\circ} \mathrm{C}$. In the produced charcoal, immediate analysis were conducted, elementary and thermal. The basic density of wood had positive correlation with $\mathrm{C} / \mathrm{H}$ relation, and negative correlation with ash, nitrogen, oxygen, sulfur contents and S/G relation. The wood contents of ash, nitrogen, sulfur and S/G had positive correlation among each other, and negative correlation with $\mathrm{C} / \mathrm{H}$ relation. In charcoal, the fixed carbon content, calorific value, elementary carbon content and $\mathrm{C} / \mathrm{H}$ relation had positive correlation among each other, and negative correlation with volatiles content. The lignin had positive correlation with gravimetric yield in charcoal (RGC) and negative correlation with fixed carbon.
\end{abstract}

Keywords: Carbonization; Eucalyptus; Lignocellulosic.

\footnotetext{
${ }^{1}$ Recebido em 21.09.2012 aceito para publicação em 30.04.2014.

${ }^{2}$ Departamento de Ciências e Linguagens, Instituto Federal de Educação, Ciência e Tecnologia de Minas Gerais, Campus Bambuí, MG, Brasil. E-mail: <vassiasoares@gmail.com>.

${ }^{3}$ Departamento de Química e Departamento de Ciências Florestais, Universidade Federal de Lavras, Lavras, MG, Brasil. E-mail: <bianchi@dqi.ufla.br>e <trugilho@dcf.ufla.br>.

${ }^{4}$ Graduando em Química, Universidade Federal de Lavras, Lavras, MG, Brasil. E-mail: <ariclenejr@gmail.com>.

${ }^{5}$ Graduação em Química, Universidade Federal de Lavras, Lavras, MG, Brasil. E-mail: <julihoefler@gmail.com>.
} 


\section{INTRODUÇÃO}

A possibilidade de mudança climática devido ao aumento da concentração de $\mathrm{CO}_{2}$ e $\mathrm{CH}_{4}$ na atmosfera causado pela queima de combustíveis fósseis e a limitação e ampla dependência de países industrializados dos combustíveis fósseis, bem como os altos preços da energia, têm resultado em aumento no interesse por energia proveniente de recursos renováveis. A biomassa, a exemplo da madeira e dos resíduos da agroindústria, se utilizada como recurso energético renovável, tem o potencial de contribuir para o futuro energético em muitos países, incluindo o Brasil.

No consumo de madeira para produção de energia, destaca-se o carvão vegetal, em decorrência da demanda do produto no setor siderúrgico. O Brasil, como grande produtor de ferro-gusa, tem alta demanda de carvão vegetal.

Um dos grandes problemas enfrentados pelas indústrias siderúrgicas é a heterogeneidade do carvão vegetal. Variações nas características desse produto são inerentes às condições do processo de carbonização e às características da madeira utilizada, como a espécie, as características químicas e a idade. Entretanto, existe a necessidade de conhecimento mais aprofundado sobre a influência da madeira sobre as características do carvão vegetal. Além disso, as reações químicas que ocorrem na madeira, durante o processo de carbonização, não estão completamente elucidadas. Dessa forma, melhorias no processo de fabricação do carvão vegetal ficam prejudicadas pela falta de conhecimentos prévios sobre o comportamento da madeira diante do aumento de temperatura em atmosfera inerte e, consequentemente, pela falta de informação sobre a influência das características da madeira sobre as características do carvão vegetal. Para madeiras destinadas à produção de carvão vegetal, deve-se procurar por materiais genéticos com menores relações $\mathrm{S} / \mathrm{G}$, uma vez que o grupo guaiacil é mais preservado no processo de pirólise. O grupo siringil possui um grupo metoxila a mais do que o guaiacil, tornandose mais reativo, ou seja, mais facilmente oxidado durante a pirólise.

A composição do carvão vegetal é diferente da composição da biomassa, sendo a variação na composição dos variados tipos de biomassa maior que a variação observada em carvões oriundos de diferentes biomassas. A biomassa é normalmente rica em umidade, compostos voláteis, $\mathrm{Ca}, \mathrm{Cl}, \mathrm{H}, \mathrm{K}, \mathrm{Mg}, \mathrm{Mn}, \mathrm{Na}$, O e $\mathrm{P}$ e possui menores teores de cinzas, carbono fixo, Al, Fe, N, Si, S e Ti, quando comparada com o carvão vegetal (VASSILEV et al., 2010). Outra relação de grande importância no processo de carbonização da madeira é a relação $\mathrm{C} / \mathrm{H}$, que tende a um maior valor absoluto no carvão quando comparado com a madeira, devido à maior aromatização e alteração química do material.

O processo de carbonização pode ser entendido ao se estudar o comportamento dos três principais componentes da madeira (celulose, hemiceluloses e lignina) diante da pirólise. Compreendendo o comportamento desses componentes, é possível compreender também como se realiza a carbonização (OLIVEIRA et al., 1982). Entretanto, analisar o comportamento da madeira sob pirólise considerando apenas o comportamento dos seus componentes principais é apenas uma aproximação grosseira do fenômeno, uma vez que as interações entre esses componentes, as quais são de grande importância na reatividade e resistência do material, não são consideradas.

A composição elementar do carvão vegetal e suas propriedades também dependem da temperatura final de carbonização. Com o aumento da temperatura, o teor de carbono fixo aumenta significativamente e ocorre diminuição no rendimento e teor de hidrogênio (DEMIRBAS, 2009). Variações no rendimento em carvão vegetal e carbono fixo são comuns e opostas. A perda de massa da fração sólida é, de certa maneira, compensada pelo aumento no teor de carbono fixo (ELYOUNSSI et al., 2010).

Elyounssi et al. (2010) relataram que o rendimento em carbono fixo aumenta até certa temperatura e, após atingir o ponto máximo, começa a diminuir. O rendimento máximo em carbono fixo encontrado corresponde a rendimentos em carvão vegetal de aproximadamente $40 \%$ a $50 \%$.

Diante do exposto, este trabalho foi realizado com o objetivo de verificar a relação entre as características da madeira de híbridos de Eucalyptus grandis x Eucalyptus urophylla e do carvão vegetal, sem levar em consideração os parâmetros da pirólise.

\section{MATERIAL E MÉTODOS}

Foi utilizada madeira de híbridos de Eucalyptus grandis $x$ Eucalyptus urophylla em três idades distintas (3, 5 e 7 anos), provenientes de plantio comercial localizado 
no Estado de São Paulo. Foram retirados discos a 1,30 $\mathrm{m}$ em relação à base da árvore. Para cada idade foram amostradas nove árvores. Para determinação da densidade básica, foram utilizadas duas cunhas opostas de cada disco. As outras duas cunhas foram empregadas para a análise química e preparo do carvão. A densidade básica da madeira foi avaliada utilizando o método de imersão, segundo a Norma NBR 11941 (ABNT, 2003). Na madeira foram realizadas as seguintes análises: extrativos segundo norma ABCP M3/69 (ATBCP, 1968), lignina Klason (GOMIDE; DEMUNER, 1986), holocelulose segundo norma TAPPI T9 wd-75 (TAPPI, 1998), celulose segundo norma TAPPI T 17 wd-70 (TAPPI, 1998), cinza segundo norma TAPPI T-15 wd-80 (TAPPI, 1998), relação siringilguaiacil (LIM; DENCE, 1992) e poder calorífico superior segundo norma NBR 8633 (ABNT, 1983). O teor de hemiceluloses foi obtido por diferença. A análise elementar (CHNS-O) foi realizada com $2,5 \mathrm{mg}( \pm 0,5)$ do material com granulometria menor que 200 mesh (madeira ou carvão), no equipamento CHNS-O Flash EA 1112 Series.

As carbonizações foram realizadas em forno elétrico adaptado com sistema de recuperação de gases. O dispositivo de entrada e saída de parâmetros foi informatizado. As cunhas de massa e tamanhos variáveis foram previamente secas em estufa a $105^{\circ} \mathrm{C}$ e pirolisadas com taxa de aquecimento de cerca de $1,67^{\circ} \mathrm{C} \mathrm{min}{ }^{-1} \mathrm{e}$ temperatura final de $450^{\circ} \mathrm{C}$. Posterior à carbonização, foram determinados os rendimentos gravimétricos em carvão, em líquido pirolenhoso e em gases não condensáveis. A análise química imediata, com o objetivo de determinar os teores de umidade, materiais voláteis (TMV), cinza (TCZ) e, por diferença, o teor de carbono fixo (TCF), oi realizada de acordo com a norma NBR 8112 (ABNT, 1986). O poder calorífico superior do carvão também foi determinado, segundo a norma 8633 da ABNT (1983).

Para análise do experimento, foi utilizado o delineamento inteiramente casualizado em três tratamentos (idades) e três repetições. Determinou-se a associação entre as características da madeira e do carvão vegetal, pelo método das correlações de Pearson, utilizando-se o software SAEG.

\section{RESULTADOS}

Os resultados médios de densidade básica e características químicas da madeira e do carvão vegetal de híbridos de Eucalyptus urophylla x Eucalyptus grandis, nas três idades diferentes, encontram-se na Tabela 1.

Tabela 1 - Valores médios da densidade básica e características químicas da madeira, rendimentos gravimétricos e características químicas do carvão.

Table 1 - Average values of basic density and chemical characteristics of wood, gravimetric yields and chemical characteristics of charcoal.

\begin{tabular}{|c|c|c|c|c|c|c|c|}
\hline \multicolumn{4}{|c|}{ Madeira } & \multicolumn{4}{|c|}{ Carvão vegetal } \\
\hline & 3 anos & 5 anos & 7 anos & & 3 anos & 5 anos & 7 anos \\
\hline $\mathrm{d}\left(\mathrm{g} \mathrm{cm}^{-3}\right)$ & 0,37 & 0,44 & 0,54 & RGC (\%) & 31,61 & 30,55 & 33,06 \\
\hline Lig* (\%) & 21,52 & 21,52 & 21,97 & RLP (\%) & 40,22 & 44,19 & 38,55 \\
\hline Extrativos (\%) & 8,26 & 9,44 & 9,43 & RGNC (\%) & 28,18 & 25,26 & 28,39 \\
\hline Holo (\%) & 73,47 & 72,60 & 72,42 & TMV (\%) & 20,08 & 18,85 & 22,82 \\
\hline Celulose (\%) & 43,58 & 42,30 & 42,70 & TCF (\%) & 78,39 & 80,29 & 76,85 \\
\hline Hemi (\%) & 29,89 & 30,30 & 29,72 & & & & \\
\hline TCZ (\%) & 0,41 & 0,31 & 0,18 & TCZ (\%) & 1,53 & 0,87 & 0,33 \\
\hline PCS (kcal kg ${ }^{-1}$ ) & 4454 & 4547 & 4385 & PCS (kcal kg-1) & 7062 & 7215 & 7193 \\
\hline C $(\%)$ & 43,97 & 44,49 & 45,60 & C $(\%)$ & 81,28 & 81,60 & 79,81 \\
\hline $\mathrm{H}^{*}(\%)$ & 6,18 & 6,18 & 6,17 & H (\%) & 3,27 & 3,29 & 3,42 \\
\hline N (\%) & 0,27 & 0,22 & 0,19 & N (\%) & 0,25 & 0,18 & 0,16 \\
\hline S (\%) & 0,38 & 0,36 & 0,29 & S (\%) & 0,04 & 0,04 & 0,02 \\
\hline O (\%) & 49,22 & 48,75 & 47,75 & O (\%) & 15,16 & 14,89 & 16,60 \\
\hline $\mathrm{C} / \mathrm{H}$ & 7,12 & 7,20 & 7,39 & $\mathrm{C} / \mathrm{H}$ & 24,84 & 24,82 & 23,43 \\
\hline $\mathrm{S} / \mathrm{G}$ & 3,50 & 3,20 & 2,30 & & & & \\
\hline
\end{tabular}

*Propriedades sem diferença significativa entre as idades: $\mathrm{d}$ = densidade básica; Lig = lignina; Holo = holocelulose; Hemi = hemiceluloses; TCZ = teor de cinzas; PCS = poder calorífico superior; S/G = relação siringil-guaiacil; RGC = rendimento gravimétrico em carvão vegetal; $\mathrm{RLP}$ = rendimento gravimétrico em líquido pirolenhoso; RGNC = rendimento gravimétrico em gases não condensáveis; TMV = teor de materiais voláteis; e TCF = teor de carbono fixo. 
As correlações entre as propriedades da madeira são apresentadas na Tabela 2. A densidade básica da madeira correlacionou-se positivamente com a relação carbono-hidrogênio e negativamente com os teores de cinzas, nitrogênio, enxofre e relação siringil-guaiacil (S/G). Os teores de cinzas, nitrogênio, enxofre e S/ $\mathrm{G}$ correlacionaram-se positivamente entre si. Essas mesmas variáveis também se correlacionaram negativamente com a variável $\mathrm{C} / \mathrm{H}$.

As correlações entre as propriedades do carvão vegetal encontram-se na Tabela 3 . O rendimento gravimétrico em carvão vegetal (RGC) correlacionou-se positivamente com o teor de materiais voláteis (TMV) e negativamente com o teor de carbono fixo (TCF) e com o rendimento em líquido pirolenhoso (RLP). O teor de carbono fixo (TCF), o poder calorífico superior (PCS), o teor de carbono elementar e a relação carbonohidrogênio $(\mathrm{C} / \mathrm{H})$ correlacionaram-se negativamente com o teor de materiais voláteis (TMV), enquanto o PCS do carvão, positivamente com a variável C/H do carvão. Os teores de oxigênio, hidrogênio e rendimento gravimétrico em carvão vegetal (RGC) apresentaram correlação negativa com a relação $\mathrm{C} / \mathrm{H}$, conforme esperado, pois as mesmas variáveis apresentaram correlação positiva com o TMV.

Tabela 2 - Correlação entre as propriedades da madeira.

Table 2 - Correlation between wood properties.

\begin{tabular}{|c|c|c|c|c|c|c|c|c|c|c|c|c|}
\hline & TET & DB & Lig & Holo & TCZ & PCS & C & $\mathrm{H}$ & $\mathrm{N}$ & $\mathrm{S}$ & $\mathrm{O}$ & $\mathrm{C} / \mathrm{H}$ \\
\hline DB & 0,54 & 1,00 & & & & & & & & & & \\
\hline Lig & $-0,07$ & 0,32 & 1,00 & & & & & & & & & \\
\hline Holo & $-0,72$ & $-0,59$ & $-0,01$ & 1,00 & & & & & & & & \\
\hline TCZ & $-0,49$ & $-0,95$ & $-0,28$ & 0,46 & 1,00 & & & & & & & \\
\hline PCS & $-0,04$ & $-0,53$ & $-0,38$ & 0,31 & 0,41 & 1,00 & & & & & & \\
\hline $\mathrm{C} 0,50$ & 0,55 & 0,16 & $-0,13$ & $-0,56$ & $-0,21$ & 1,00 & & & & & & \\
\hline $\mathrm{HO}, 21$ & $-0,20$ & $-0,31$ & 0,25 & 0,17 & 0,47 & 0,62 & 1,00 & & & & & \\
\hline $\mathrm{N}-0,44$ & $-0,75$ & $-0,16$ & 0,31 & 0,91 & 0,11 & $-0,43$ & 0,13 & 1,00 & & & & \\
\hline S- 0,08 & $-0,71$ & $-0,47$ & 0,05 & 0,77 & 0,65 & $-0,53$ & 0,26 & 0,66 & 1,00 & & & \\
\hline $\mathrm{O}-0,49$ & $-0,48$ & $-0,12$ & 0,11 & 0,49 & 0,15 & $-1,00$ & $-0,69$ & 0,35 & 0,44 & 1,00 & & \\
\hline $\mathrm{C} / \mathrm{H}$ & 0,52 & 0,75 & 0,34 & $-0,24$ & $-0,75$ & $-0,44$ & 0,94 & 0,33 & $-0,57$ & $-0,74$ & $-0,91$ & 1,00 \\
\hline$S / G$ & $-0,51$ & $-0,97$ & $-0,30$ & 0,53 & 0,94 & 0,59 & $-0,66$ & 0,08 & 0,72 & 0,74 & 0,60 & $-0,83$ \\
\hline
\end{tabular}

TET = teor de extrativos totais; DB = densidade básica; Lig = teor de lignina; Holo = teor de holocelulose; TCZ = teor de cinzas; PCS = poder calorífico superior; $\mathrm{C}=$ teor de carbono; $\mathrm{H}=$ teor de hidrogênio; $\mathrm{N}=$ teor de nitrogênio; $\mathrm{S}=$ teor de enxofre; $\mathrm{O}=$ teor de oxigênio; $\mathrm{C} / \mathrm{H}=$ relação entre os teores de carbono e hidrogênio; e S/G relação entre os teores de lignina siringil e guaiacil.

Tabela 3 - Correlação entre as propriedades do carvão vegetal.

Table 3 - Correlation between charcoal properties.

\begin{tabular}{|c|c|c|c|c|c|c|c|c|c|c|c|c|c|}
\hline & RGC & RLP & RGNC & TMV & TCZ & TCF & PCS & $\mathrm{C}$ & $\mathrm{H}$ & $\mathrm{N}$ & $\mathrm{S}$ & $\mathrm{O}$ & $\mathrm{C} / \mathrm{H}$ \\
\hline RGC & 1,00 & & & & & & & & & & & & \\
\hline RLP & $-0,71$ & 1,00 & & & & & & & & & & & \\
\hline RGNC & 0,34 & $-0,90$ & 1,00 & & & & & & & & & & \\
\hline TMV & 0,95 & $-0,56$ & 0,17 & 1,00 & & & & & & & & & \\
\hline TCZ & $-0,34$ & 0,16 & 0,00 & $-0,34$ & 1,00 & & & & & & & & \\
\hline TCF & $-0,94$ & 0,56 & $-0,18$ & $-0,99$ & 0,17 & 1,00 & & & & & & & \\
\hline PCS & $-0,65$ & 0,34 & $-0,05$ & $-0,72$ & $-0,26$ & 0,80 & 1,00 & & & & & & \\
\hline $\mathrm{C}$ & $-0,82$ & 0,23 & 0,20 & $-0,91$ & 0,29 & 0,90 & 0,64 & 1,00 & & & & & \\
\hline $\mathrm{H}$ & 0,80 & $-0,66$ & 0,40 & 0,74 & $-0,39$ & $-0,71$ & $-0,60$ & $-0,54$ & 1,00 & & & & \\
\hline $\mathrm{N}$ & $-0,14$ & $-0,01$ & 0,11 & $-0,22$ & 0,89 & 0,06 & $-0,24$ & 0,22 & $-0,31$ & 1,00 & & & \\
\hline S & $-0,37$ & 0,32 & $-0,21$ & $-0,20$ & 0,57 & 0,11 & $-0,19$ & 0,15 & $-0,19$ & 0,40 & 1,00 & & \\
\hline $\mathrm{O}$ & 0,79 & $-0,18$ & $-0,24$ & 0,89 & $-0,30$ & $-0,87$ & $-0,61$ & $-1,00$ & 0,49 & $-0,23$ & $-0,16$ & 1,00 & \\
\hline $\mathrm{C} / \mathrm{H}$ & $-0,91$ & 0,56 & $-0,20$ & $-0,90$ & 0,40 & 0,87 & 0,69 & 0,80 & $-0,94$ & 0,30 & 0,21 & $-0,76$ & 1,00 \\
\hline
\end{tabular}

RGC = rendimento gravimétrico em carvão vegetal; RLP = rendimento em líquido pirolenhoso; RGNC = rendimento em gases não condensáveis; TMV = teor de materiais voláteis; TCZ = teor de cinzas; $\mathrm{TCF}=$ teor de carbono fixo; PCS = poder calorífico superior; $\mathrm{C}=$ teor de carbono; $\mathrm{H}$ = teor de hidrogênio; $\mathrm{N}$ = teor de nitrogênio; $\mathrm{S}=$ teor de enxofre; $\mathrm{O}=$ teor de oxigênio; e $\mathrm{C} / \mathrm{H}=$ relação entre os teores de carbono e hidrogênio.

Revista Árvore, Viçosa-MG, v.38, n.3, p.543-549, 2014 
As correlações entre as propriedades do carvão vegetal e da madeira estão apresentadas na Tabela 4. Conforme esperado, observou-se correlação positiva no teor de cinzas na madeira e no carvão vegetal, visto que esse componente não é afetado significativamente durante a pirólise. A relação C/H no carvão vegetal correlacionou-se negativamente com a relação $\mathrm{C} / \mathrm{H}$ da madeira. Vale ressaltar que houve correlação negativa entre o teor de carbono elementar no carvão vegetal e os teores de carbono elementar e $\mathrm{C} / \mathrm{H}$ da madeira. O poder calorífico superior da madeira (PCS) apresentou correlação positiva $(0,70)$ com o rendimento em líquido pirolenhoso (RLP) e correlação negativa $(0,75)$ com o rendimento gravimétrico em carvão vegetal (RGC).

\section{DISCUSSÃO}

\subsection{Correlação entre as propriedades da madeira}

Os elementos químicos constituintes da cinza, incluindo o enxofre e o nitrogênio, são requeridos em maior quantidade nos estágios iniciais do desenvolvimento da planta. Conforme observado na Tabela 1, a densidade básica do material lenhoso aumentou de acordo com a idade da árvore. Dessa forma, a madeira de maior densidade básica, por ser mais madura, apresentou menores teores de cinza e nitrogênio. Outra variável que apresentou comportamento semelhante, quando correlacionada com a densidade básica, foi a relação S/G. O material mais jovem, de menor densidade básica, apresentou maior proporção de grupo siringil.
A correlação negativa entre as relações S/G e C/ $\mathrm{H}$ pode ser explicada pela estrutura química das unidades siringil e guaiacil. O grupo siringil apresenta fórmula molecular $\mathrm{C}_{11} \mathrm{H}_{14} \mathrm{O}_{4}$, que pode ser convertida na fórmula percentual $\mathrm{C}_{62,9 \%} \mathrm{H}_{6,7 \%} \mathrm{O}_{30,5 \%}$. Esse fórmula expressa a proporção da massa de cada elemento em relação à massa total da molécula. O grupo guaiacil apresenta fórmulas molecular e percentual iguais a $\mathrm{C}_{10} \mathrm{H}_{12} \mathrm{O}_{3} \mathrm{e}$ $\mathrm{C}_{66,7 \%} \mathrm{H}_{6,7 \%} \mathrm{O}_{26,7 \%}$, respectivamente. Como a relação C/ $\mathrm{H}$ é obtida a partir de valores percentuais de carbono e hidrogênio, pode-se presumir que a relação $\mathrm{C} / \mathrm{H}$ seria, aproximadamente, 9,4 para uma molécula de siringil e 10,0 para uma molécula de guaiacil. Dessa forma, maior relação S/G resulta em menor relação $\mathrm{C} / \mathrm{H}$.

A alta correlação positiva entre os teores de carbono e oxigênio ocorreu pelo fato de o teor de oxigênio ter sido obtido por diferença e o elemento presente, em maior proporção, ser o carbono.

\subsection{Correlação entre as propriedades do carvão vegetal}

A correlação negativa entre RGC e TCF também foi encontrada por Oliveira et al. (2010) e Vale et al. (2001). Vale et al. (2001) sugeriram, ainda, que a diminuição do teor de carbono fixo com o aumento do rendimento em carvão vegetal resultaria da presença de compostos oriundos dos extrativos, a ponto de aumentar, juntamente com a lignina, o rendimento em carvão vegetal, causado pelo incremento dos teores de seu material volátil.

Tabela 4 - Correlação entre as propriedades da madeira e do carvão vegetal.

Table 4 - Correlation between wood and charcoal properties.

\begin{tabular}{ccccccccccccccc}
\hline & & \multicolumn{11}{c}{ Madeira } \\
\cline { 2 - 15 } & TET & DB & Lig & Holo & TCZ & PCS & C & H & N & S & O & C/H & S/G \\
\hline \multirow{6}{*}{ Carvão } & RGC & 0,08 & 0,46 & 0,47 & 0,07 & $-0,34$ & $\mathbf{- 0 , 7 5}$ & 0,57 & $-0,01$ & $-0,02$ & $-0,64$ & $-0,54$ & 0,68 & $-0,55$ \\
& RLP & 0,45 & $-0,29$ & $-0,46$ & $-0,32$ & 0,31 & $\mathbf{0 , 7 0}$ & $-0,31$ & 0,18 & 0,12 & 0,73 & 0,26 & $-0,45$ & 0,41 \\
& RGNC & $-0,66$ & 0,10 & 0,33 & 0,39 & $-0,20$ & $-0,47$ & 0,07 & $-0,23$ & $-0,15$ & $-0,59$ & $-0,02$ & 0,18 & $-0,21$ \\
& TMV & 0,17 & 0,39 & 0,35 & 0,08 & $-0,25$ & $-0,59$ & 0,68 & 0,24 & 0,06 & $-0,52$ & $-0,68$ & $\mathbf{0 , 7 2}$ & $-0,47$ \\
& TCZ & $-0,60$ & $\mathbf{- 0 , 9 3}$ & $-0,32$ & 0,48 & $\mathbf{0 , 9 3}$ & 0,24 & $-0,69$ & $-0,07$ & $\mathbf{0 , 8 1}$ & 0,63 & 0,64 & $\mathbf{- 0 , 8 0}$ & $\mathbf{0 , 9 1}$ \\
& TCF & $-0,07$ & $-0,24$ & $-0,31$ & $-0,17$ & 0,10 & 0,57 & $-0,59$ & $-0,23$ & $-0,21$ & 0,44 & 0,59 & $-0,61$ & 0,33 \\
& PCS & 0,14 & 0,26 & $-0,29$ & $-0,52$ & $-0,33$ & 0,22 & $-0,26$ & $-0,21$ & $-0,45$ & 0,17 & 0,28 & $-0,22$ & $-0,20$ \\
& C & $-0,46$ & $-0,35$ & $-0,12$ & 0,15 & 0,17 & 0,49 & $-0,63$ & $-0,29$ & $-0,13$ & 0,28 & 0,64 & $-0,63$ & 0,42 \\
& H & 0,14 & 0,44 & 0,64 & 0,03 & $-0,44$ & $-0,65$ & 0,57 & $-0,10$ & $-0,28$ & $-0,82$ & $-0,51$ & $\mathbf{0 , 7 2}$ & $-0,49$ \\
& S & $-0,23$ & $-0,57$ & $-0,59$ & 0,13 & 0,59 & 0,28 & $-0,32$ & 0,12 & 0,47 & 0,36 & 0,28 & $-0,44$ & 0,63 \\
& O & 0,48 & 0,35 & 0,08 & $-0,16$ & $-0,17$ & $-0,46$ & 0,63 & 0,32 & 0,13 & $-0,24$ & $-0,64$ & 0,62 & $-0,42$ \\
& C/H & $-0,28$ & $-0,47$ & $-0,54$ & 0,04 & 0,39 & 0,67 & $-0,64$ & $-0,02$ & 0,14 & $\mathbf{0 , 7 0}$ & 0,61 & $\mathbf{- 0 , 7 6}$ & 0,52 \\
\hline
\end{tabular}

TET = teor de extrativos totais; DB = densidade básica; Lig = teor de lignina; Holo = teor de holocelulose; TCZ = teor de cinzas; PCS = poder calorífico superior; $\mathrm{C}=$ teor de carbono; $\mathrm{H}=$ teor de hidrogênio; $\mathrm{N}=$ teor de nitrogênio; $\mathrm{S}=$ teor de enxofre; $\mathrm{O}=$ teor de oxigênio; $\mathrm{C} / \mathrm{H}$ = relação entre os teores de carbono e hidrogênio; S/G relação entre os teores de lignina siringil e guaiacil; RGC = rendimento gravimétrico em carvão vegetal; RLP = rendimento em líquido pirolenhoso; RGNC = rendimento em gases não condensáveis; TMV = teor de materiais voláteis; TCZ = teor de cinzas; TCF = teor de carbono fixo; e PCS = poder calorífico superior. 
Silvério et al. (2006), ao analisarem amostras de extrativos de híbridos de Eucalyptus urophylla $x$ Eucalyptus grandis por espectroscopia vibracional na região do infravermelho, observaram a presença de compostos oxigenados, como ácidos carboxílicos, ésteres de ácidos graxos, compostos fenólicos e alcoóis. Neste estudo, foi encontrada correlação positiva entre o teor de materiais voláteis e os teores de oxigênio e hidrogênio (0,89 e 0,74, respectivamente). Conforme explanação anterior, o TMV provém de componentes oriundos dos extrativos e, devido à presença de extrativos oxigenados, foi observado aumento no teor de materiais voláteis com o aumento no teor de oxigênio do material.

Maior teor de carbono leva, obviamente, à maior relação $\mathrm{C} / \mathrm{H}$. Parte do teor de carbono encontrado no carvão vegetal é atribuída aos materiais voláteis, que também possuem carbono em sua composição. Assim, o incremento no teor de carbono fixo com o aumento no teor de carbono elementar sugere quantidade maior de carbono elementar dificilmente pirolisável. Entre os tipos de estruturas carbônicas dificilmente pirolisadas presentes na madeira e, portanto, no carvão vegetal, encontram-se as cadeias aromáticas. Portanto, aumento de compostos aromáticos, ou seja, que possuem maior relação $\mathrm{C} / \mathrm{H}$, eleva o teor de carbono fixo do carvão vegetal.

Com relação à correlação positiva entre o poder calorífico superior (PCS) e a relação C/H, pode-se inferir que o aumento da relação $\mathrm{C} / \mathrm{H}$ está diretamente ligado à maior aromaticidade do material e, portanto, à maior quantidade de ligações $C=C$. A energia de ligação entre dois carbonos é da ordem de $348 \mathrm{~kJ} \mathrm{~mol}^{-1}$ para ligações C-C e $518 \mathrm{~kJ} \mathrm{~mol}^{-1}$ para ligações C = C, em compostos aromáticos (ATKINS; JONES, 2006). As ligações químicas que possuem maior energia de ligação, quando rompidas, liberam mais energia e, consequentemente, caracterizam um material com maior poder calorífico.

\subsection{Correlação entre as propriedades da madeira $x$ carvão vegetal}

Pode-se dizer que a quantidade de carbono elementar presente no carvão vegetal não esteja relacionada com a quantidade de carbono elementar presente na madeira e, sim, com o tipo de estrutura química presente na madeira, visto que a relação $\mathrm{C} / \mathrm{H}$ da madeira e do carvão correlacionou-se negativamente.

Revista Árvore, Viçosa-MG, v.38, n.3, p.543-549, 2014
A correlação positiva entre PCS da madeira e RLP, bem como a correlação negativa entre o PCS da madeira e o RGC, permite inferir que parte das substâncias responsáveis pelo poder calorífico da madeira compõe o líquido pirolenhoso. No rol das substâncias constituintes do líquido pirolenhoso estão derivados da lignina e extrativos. Dessa forma, maior rendimento gravimétrico em carvão vegetal poderia ser alcançado pela presença, em maior quantidade, de componentes originários da lignina e dos extrativos, durante a pirólise.

\section{CONCLUSÃO}

Pelos resultados, pode-se concluir que:

a) A densidade básica da madeira correlacionase positivamente com a relação carbono/hidrogênio e negativamente com os teores de cinza, nitrogênio, oxigênio, enxofre e relação siringil/guaiacil.

b) Os teores de cinza, nitrogênio, enxofre e siringil/ guaiacil da madeira correlacionam-se positivamente entre si e negativamente com a variável carbono/ hidrogênio da madeira.

c) O rendimento gravimétrico em carvão correlaciona-se positivamente com o teor de materiais voláteis e negativamente com o teor de carbono fixo e rendimento em líquido pirolenhoso.

d) O teor de carbono fixo, o poder calorífico do carvão vegetal, o teor de carbono e a relação carbono/ hidrogênio correlacionam-se positivamente entre si e negativamente com o teor de materiais voláteis.

e) Os teores de oxigênio, hidrogênio e rendimento gravimétrico em carvão apresentam correlação negativa com a relação carbono/hidrogênio.

f) Há correlação positiva entre os teores de cinza da madeira e do carvão vegetal.

g) A relação carbono/hidrogênio do carvão vegetal correlaciona-se negativamente com a relação carbono/ hidrogênio da madeira e o teor de lignina.

h) Há correlação negativa entre o teor de carbono do carvão vegetal e os teores de lignina, carbono e carbono/hidrogênio da madeira.

\section{AGRADECIMENTOS}

A CAPES, CNPq, FAPEMIG e LCP - UFV, pelo apoio.

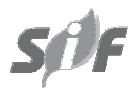




\section{REFERÊNCIAS}

ASSOCIAÇÃO BRASILEIRA DE NORMAS TÉCNICAS - ABNT. NBR 8112: carvão vegetal: análise imediata. Rio de Janeiro: 1986. 8p.

ASSOCIAÇÃO BRASILEIRA DE NORMAS TÉCNICAS - ABNT. NBR 8633: carvão vegetal: determinação do poder calorífico. Rio de Janeiro: 1983. 13p.

\section{ASSOCIAÇÃOBRASILEIRADE NORMAS TÉCNICAS - ABNT. BR 11941: madeira, determinação da densidade básica. Rio de Janeiro: 2003. 6p.}

ASSOCIAÇÃO TÉCNICA BRASILEIRA DE CELULOSE E PAPEL - ATBCP. Normas de ensaio. São Paulo: 1968. 6p.

ATKINS, P.; JONES, L. Princípios de química: questionando a vida moderna e o meio ambiente. 3.ed. Porto Alegre: Bookman, 2006. 968p.

DEMIRBAS, A. Biorefineries: current activities and future developments. Energy

Conversion and Management, v.50, n.11, p.2782-2801, 2009.

ELYOUNSSI, K.; BLIN, J. L.; HALIM, M. Highyield charcoal production by two-step pyrolysis. Journal of Analytical and Applied Pyrolysis, v.87, n.1, p.138-143, 2010.

GOMIDE, J. L.; DEMUNER, B. J. Determinação do teor de lignina em material lenhoso: método Klason modificado. O Papel, v.47, n.8, p.36-38, 1986.
LIM, S. Y.; DENCE, C. W. Methods in lignin chemistry. Berlin: Springer-Verlag, 1992. 578p.

OLIVEIRA, J. B.; GOMES, P. A.; ALMEIDA, M. R. Caracterização e otimização do processo de fabricação de carvão vegetal em fornos de alvenaria. In: FUNDAÇÃO CENTRO TECNOLÓGICO DE MINAS GERAIS. Carvão vegetal: destilação, carvoejamento, propriedades, controle de qualidade. Belo Horizonte: 1982. p.62-102. (Série de Publicações Técnicas).

OLIVEIRA, A. C. et al. Parâmetros de qualidade da madeira e do carvão vegetal de Eucalyptus pellita F. Muell. Scientia Forestalis, v.38, n.87, p.431-439, 2010.

SILVÉRIO, F. O. et al. Metodologia de extração e determinação do teor de extrativos em madeiras de eucalipto. Revista Árvore, v.30, n.6, p.10091016, 2006.

\section{TAPPI TECHNICAL DIVISIONS AND COMMITTEES. TAPPI test methods. Atlanta: 1998. 460p.}

VALE, A. T. et al. Relações entre a densidade básica da madeira, o rendimento e a qualidade do carvão vegetal de espécies do cerrado. Revista Árvore, v.25, n.1, p.89-95, 2001.

VASSILEV, S. V. et al. An overview of the chemical composition of biomass. Fuel, v.89, n.5, p.913-933, 2010 